\title{
Simulation of Magnetohydrodynamic Effects on an Ionised Hypersonic Flow by Using the TAU Code
}

\author{
Carmen Böttcher ${ }^{1}$, Volker Hannemann ${ }^{2}$, Heinrich Lüdeke ${ }^{1}$ \\ 1 DLR, Institute of Aerodynamics and Flow Technology, \\ Lilienthalplatz 7, 38018 Braunschweig, Germany \\ 2 DLR, Institute of Aerodynamics and Flow Technology, \\ Bunsenstraße 10, 37073 Göttingen, Germany
}

\begin{abstract}
Summary
In the present investigation the DLR TAU code is extended to support future experimental investigations of magnetohydrodynamic effects in high temperature hypersonic flows. According to the conditions in the High Enthalpy Shock Tunnel Göttingen (HEG) the first steps in enhancing the TAU code are the implementation of a source term formulation of electromagnetic forces and the calculation of the electrical conductivity of air as a gas mixture in chemical non equilibrium. To verify the source term implementation a perfect gas study related to numerical simulations from Poggie and Gaitonde is conducted and shows reasonable agreement. Applied to the experimental conditions the model predicts a noticeable increase of the shock stand off distance.
\end{abstract}

\section{Nomenclature}

$\begin{array}{llll}\overline{\bar{\tau}} & \text { total stress tensor } & \boldsymbol{B} & \text { magnetic induction vector } \\ E_{t} & \text { total energy } & \boldsymbol{E} & \text { electric field vector } \\ q & \text { heat flux term } & \sigma & \text { electrical conductivity } \\ \boldsymbol{r} & \text { position vector } & \mu_{0} & \text { magnetic permeability } \\ \boldsymbol{j} & \text { electric current density } & \boldsymbol{m} & \text { dipole moment }\end{array}$

\section{Introduction}

Due to high temperatures between shock and body at hypersonic flights a high level of natural ionisation is generated. About 50 years ago first ideas came up to influence ionised flows with magnetic fields. The theory of the interaction of an electrically conducting fluid with electric and magnetic fields, called magnetohydrodynamics (MHD), was founded. In the present study an extension of the Navier-Stokes equations for the simulation of the influence of magnetic fields on an ionised hypersonic flow will be described. As a tool for the solution of the Reynolds averaged Navier-Stokes equations the DLR TAU code for hypersonic flows $[4,5]$ is used. It provides a numerical support for HEG experiments as shown in numerous former projects. The code is well validated in terms of hypersonic 